\title{
Prof. Dr. Rosemarie Will, Humboldt-Universität zu Berlin
}

\author{
Juristinnen machen Karriere - wir stellen sie vor
}

Das Interview führte Anke Gimbal, Geschäftsführerin, Deutscher Juristinnenbund e.V. (djb), am 22. August 2014 in Berlin.

\begin{abstract}
Wie verbrachten Sie Ihre Schulzeit in Kamenz. War es selbstverständlich, dass Sie Abitur machen und studieren?

Für mich war es relativ selbstverständlich, dass ich Abitur mache und studiere. Ich hatte das Glück, nicht an Bedingungen gebunden zu sein, weil ich nämlich in die Kinder- und Jugendsportschule ging. Damit war schon die Entscheidung getroffen, dass ich zum Abiturlehrgang gehörte. Es ging strikt nach Leistung, andere Dinge wurden ausgeblendet. Doch nachdem ich gesehen habe, dass ich nicht wirklich jemand Großes im Sport werden würde, habe ich schon nach dem Übergang zur Abiturstufe gesagt, dass ich aufhören möchte. Sie haben sich bemüht, mich anderweitig unterzubringen.
\end{abstract}

\section{Welchen Sport haben Sie denn betrieben?}

Ich war Turnerin. Für die Nationalmannschaft im Turnen wurden nur fünf Turnerinnen und eine Ersatzturnerin gebraucht. $\mathrm{Zu}$ denen hätte ich nicht gehört, das war nach dem Übergang zur Frauenklasse klar. Ich hätte bleiben können, das war keine Frage, aber ich habe aufgehört. Mein Vater fand, dass man nicht 13 Jahre lang Abitur machen muss, sondern 12 Jahre ausreichen. Ich bin in eine normale $\mathrm{EOS}^{1}$ gewechselt.

\section{Was sagte Ihre Familie zu Ihrem Wunsch, Jura zu studieren?}

Die Familie hatte überhaupt keine Meinung dazu, dass ich Jura studieren wollte. Es gab keine Juristen in meiner Familie. Ich bin also die Erste. Diese Berufswahl ist eher von Ahnungslosigkeit und Naivität geprägt worden, als von größerer Einsicht oder Wissen um das Berufsbild und die Bedingungen in der DDR. Lehrerin wollte ich nicht werden. Medizin hat mich geekelt. Also musste mir was anderes einfallen. Jura war in der DDR etwas Exklusives. Und man hat mir nachgesagt, ich hätte einen unerschütterlichen Hang zur Gerechtigkeit und würde immer alles bis zum Letzten ausdiskutieren wollen. Vielleicht war es das, aber ich glaube es nicht mal. Aber ich habe das Jurastudium durchgezogen und bin erst später darauf gekommen, was es bedeutet, in der DDR Juristin zu werden. Auch das Wissen, dass man eigentlich keine Chance hat, Rechtsanwältin zu werden, hatte ich nicht, als ich anfing. Ich war ziemlich gut in der Schule und glaubte, alles zu können. Ich war ganz ehrgeizig, aber eben auch furchtbar naiv.

\section{Wie bekam man einen Studienplatz?}

Ich habe mich noch regulär an der Uni beworben, und zwar in Leipzig, weil meine Schulfreundin dort Medizin studieren wollte. Ich dachte, wir gehen da zusammen hin. Dann kam die Hochschul-

1 Die „Erweiterte allgemeinbildende polytechnische Oberschule“ (EOS) war die höhere Schule im Schulsystem der DDR und schloss nach der zwölften Klasse mit der Hochschulreife ab.
Rosemarie Will, geb. 1949 in Bernsdorf, studierte nach dem Abitur in Kamenz von 1969 bis 1973 Rechtswissenschaft an der Humboldt-Universität zu Berlin. Nach ihrem Abschluss arbeitete sie dort ein Jahr als Forschungsstudentin, danach wurde sie als Assistentin eingestellt. 1977 folgte die Promotion mit der Arbeit „Studien zum Kampf der Arbeiterklasse um soziale Grundrechte im Kapitalismus, unter besonderer Berücksichtigung der BRD“. 1979/1980 hielt sie sich zu einem Studienaufenthalt in der Sowjetunion an der Universität Lwiw (Lemberg) auf. Von 1980 bis 1983 war sie an der Akademie der Wissenschaften der DDR im Institut für Staats- und Rechtstheorie tätig. 1983 habilitierte sich Rosemarie Will an der Humboldt-Universität zu Berlin für Öffentliches Recht mit der Arbeit „Studie über die Rolle des Staates in der politischen Organisation der sozialistischen Gesellschaft" und wurde 1984 zur Hochschuldozentin berufen.

Im September 1989 wurde sie ordentliche Professorin für Staatsrecht an der Humboldt-Universität. Von 1990 bis 1993 war sie Dekanin des Fachbereichs Rechtswissenschaft und leitete in dieser Funktion die Neuorganisation der rechtswissenschaftlichen Fakultät. Seit 1993 ist sie nach Bewerbung, Evaluation und Neuberufung auch nach dem Hochschulrecht der Bundesrepublik Professorin für Öffentliches Recht, Staatslehre und Rechtstheorie. Von 1993 bis 1995 arbeitete sie als wissenschaftliche Mitarbeiterin am Bundesverfassungsgericht im Dezernat von Prof. Dr. Dieter Grimm. Von 1996 bis 2006 war sie Richterin am Verfassungsgericht des Landes Brandenburg. Forschungsaufenthalte führten sie an die Juristischen Fakultäten der Universität Basel (1997/98) und der Staatlichen Universität Sankt Petersburg (2010).

Rosemarie Will war als Verfassungsexpertin Mitglied der Arbeitsgruppe Neue Verfassung des Runden Tisch der DDR. Aus der SED trat sie 1989 aus. 1994 wurde sie Mitglied der SPD und 1996 Mitglied der SPD-Grundwertekommission. Seit 1991 ist sie Mitglied der Humanistischen Union und war von 2005 bis 2013 deren Vorsitzende. Sie ist Mitherausgeberin der politisch-wissenschaftlichen Monatszeitschrift Blätter für deutsche und internationale Politik.

Rosemarie Will ist seit 1995 Mitglied des djb. In ihren Forschungen befasste sie sich immer wieder mit Fragen der rechtsstaatlichen Transformation von Personen und Institutionen der ehemaligen DDR. Im djb hatte sie von 2007 bis 2011 die wissenschaftliche Leitung des vom Ministerium der Justiz des Landes Brandenburg geförderten Projektes „Juristinnen in der DDR“ inne.

reform dazwischen mit den Spezialisierungen der Ausbildungsrichtungen zwischen den DDR-Fakultäten. Das bedeutete, dass in Halle und Leipzig Wirtschaftsjuristen ausgebildet wurden und nur noch in Berlin für die Justiz als Ganzes. Später kam dann noch Jena für die Staatsanwaltschaft dazu. Wieder naiv hatte ich die Vorstellung, dass ich ein richtiger Jurist in der Justiz werden will, und bin deshalb nach 
Berlin gegangen. Aber Berlin war wirklich ein Glücksgriff und viel weniger Provinz als Leipzig. Ich hatte weiter keine Schwierigkeiten, sondern nur das Problem, dass ich nach Abschluss des Studiums zu jung gewesen wäre, um in der Justiz anzufangen. Also wurde ich zwar zugelassen und für das folgende Jahr immatrikuliert, musste bis dahin aber erst einmal ein Jahr arbeiten. Die Mädchen mussten ein Jahr arbeiten, die Jungen gingen zur NVA.

Weil ich auf der Sportschule war, habe ich mir eine Arbeit im Sportumfeld gesucht. Vertretung für Sportlehrer an einer Oberschule ging nicht, da ich keine Ausbildung dafür hatte. Aber ich wurde als Pionierleiter eingestellt. Die mussten zwar eigentlich auch Grundschullehrer sein, aber es wurde nicht so scharf nachgeprüft. Ich war an einer normalen Oberschule in Kamenz und habe Sport und manchmal auch andere Fächer vertreten.

\section{Wie haben Sie das Studium in Berlin erlebt? Wie war das Studi- um aufgebaut - wo liegen die Unterschiede zu heute?}

Das Studium war ganz schulmäßig aufgebaut. Es wurde erwartet, dass man jede vorgeschriebene Vorlesung und jedes Seminar besucht. Das habe ich auch weitgehend widerstandslos und eifrig gemacht. Das Studium war viel stärker gesellschaftswissenschaftlich orientiert, also sehr ideologisch nach dem Marxismus-Leninismus ausgerichtet. Das eigentliche Fachstudium war schmal, weil z.B. diese große dogmatische Breite, die wir heute haben, im Öffentlichen Recht völlig fehlte. Es gab sie im Strafrecht und auch im Zivilrecht nur zum Teil. Insofern konnte man das Studium tatsächlich auch viel schneller - in vier Jahren absolvieren. Heute erwarten wir von den Studenten, dass sie schon wissen wo es lang geht, sich selbst orientieren, ihre Freundeskreise finden und alles richtig timen. In der DDR war dies den Studenten abgenommen. Sie kamen in eine Maschinerie, die ihre ideologischen Gründe, aber auch ihre Rationalismen hatte.

Die maximale Vorlesungsgröße war 200. Man hatte aber immer acht bis zehn kleine Gruppe von 20 bis 25 Leuten, in denen man arbeitete. Sie war wie eine Schulklasse und blieb vom ersten bis zum letzten Studienjahr gleich. Es gab manche, die den Wechsel in eine andere Gruppe beantragten. Aber sonst bewegte man sich in einem sehr festen Rahmen. Jeder kannte jeden. Das brachte viel Stabilität, war natürlich aber auch dem Umstand geschuldet, dass man die Leute sehr übersichtlich, sehr im Fokus haben und wissen wollte, wer ist wer. Wenn die Staatssicherheit etwas wissen wollte, hat sie es tatsächlich auch wissen können.

\section{Gab es ein Leben neben dem Studium und wie sah es aus?}

$\mathrm{Ja}$, es gab viel Leben. Wer nach Berlin kam, war jung, hat sich verliebt und hat sonst was gemacht außer studiert. Ich habe 1969, in der Endphase Ulbrichts und dem Übergang zu Honecker angefangen zu studieren. Die Honecker-Phase begann damit, dass er kulturell die Parole ausgab, lasst alle Blumen blühen, was er später wieder abgewürgt hat. Meine Abschlussfeier nach der Ausgabe der Diplom-Zeugnisse begann im Deutschen Theater mit einer Vorführung der „Neuen Leiden des jungen W.“ - das war der „DDR-Fänger im Roggen“ - in einer grandiosen Besetzung. Dann sind wir in die Bar hinter dem Friedrichstadt-Palast gestolpert und haben bis früh um drei nach der Fischer-Band gesungen und getanzt.
Kulturell war das ganz aufregend. Später gab es mit der BiermannAusweisung und den ganzen Repressalien einen Rückschlag. Aber zunächst einmal habe ich die großen Theater Berlins alle gesehen und erlebt, wie die ganze Kulturszene angefangen hat aufzubrechen. Wir haben es genossen und eingesogen, damals in Berlin.

\section{Was konnte man mit dem Abschluss anfangen?}

Alle Absolventen der DDR waren Diplom-Juristen. Die DDR hat zwar den Versuch gemacht, durch Sparten - in Halle und Leipzig Wirtschaftsjuristen, Jena Staatsanwälte und Berlin Justiz - eine Spezialisierung während des Studiums einzuführen. Aber es gab keine unterschiedlichen Zeugnisse, sondern alle bekamen Staatsexamen und Diplom. Theoretisch gab es daher die Möglichkeit zu wechseln. Praktisch war dies durch die Lenkungsverordnung, der auch die Juristen unterfielen, ausgeschlossen. Alle DDRAbsolventen wurden nach ihrem Abschluss staatlicherseits in Berufe gelenkt: die Berliner Absolventen in die Justiz. Darauf schauten in Berlin schon das Ministerium der Justiz, in Jena der Generalstaatsanwalt bzw. die Staatsanwälte und die Absolventen aus Leipzig und Halle gingen in die Wirtschaft. Die Familie musste schon sehr einflussreich sein, wenn sie einen aus diesen zentralen staatlichen Lenkungen rausholen konnte.

Ich habe mir angeschaut, wie gelenkt wird. Mir war während des Studiums schon klar geworden, dass ich überhaupt keine Chance hätte, Rechtsanwältin zu werden und stattdessen vermutlich Richterin in Perleberg werden würde. Das wollte ich aber nicht, sondern ich wollte in Berlin bleiben. Da ich nicht aus Berlin komme, hatte ich keine Chance dort Richterin zu werden. Die einzige Möglichkeit, in Berlin zu bleiben, war als Forschungsstudentin. Um Forschungsstudentin zu werden, musste man unter den Top 10 der Absolventen landen. Es ging nach Leistung, wobei man natürlich nicht ideologisch aufgefallen sein durfte. Das war klar. Es wurde eine bestimmte ideologische Konformität erwartet. Also habe ich mir gesagt, jetzt wirfst du die Kurbel an und versuchst, zu diesen Auserwählten zu gehören. Das hat geklappt, und ich konnte nach dem Studium promovieren.

\section{Hatten Sie Vorbilder?}

Ich habe die drei großen Professorinnen an unserer Fakultät sehr bewundert: Vera Thiel, Anita Grandke und auch Edith Oeser. Sie waren schon strahlende Erscheinungen, sehr toughe Frauen, die alle drei in ihrem Fach ziemlich überragend waren.

Gemessen an westdeutschen Verhältnissen waren drei Juraprofessorinnen viel. Aber verglichen mit der Anzahl der Studentinnen - leicht mehr Studentinnen als Studenten - und der Anzahl der Doktorandinnen - fast noch ein Gleichgewicht - waren es nicht viele. Auch in der DDR wurden es weniger Frauen je höher man in der Pyramide kam.

\section{Hatten Sie damals schon Familie, ließ sich das mit dem Studium vereinbaren?}

Mein Mann war eine Studentenliebe. Wir haben uns bei den Eingangsfeten des Studiums kennen gelernt, unser Sohn wurde ein Jahr später, 1970, geboren und wir haben geheiratet. Es ließ sich gut vereinbaren und passte sehr gut, während des Studiums ein 
Kind zu bekommen. Später tat mir leid, dass ich das zweite Kind erst nach 14 Jahren, nach Promotion und Habilitation bekommen habe. Denn auch da hatten wir Glück. Honecker förderte ganz stark Familien, auch Studentenfamilien und Kinder. Kindergarten- und Krippenplätze sowie Kindergeld wurden aufgestockt. Es gab auch an der Uni Maßnahmen für Mutter und Kind. Gemessen an heute war es nahezu ein Idealzustand. Mein Mann und ich mussten noch aus dem Studentenwohnheim ausziehen, aber ein halbes Jahr später bekamen junge Familien schon eine Familienwohnung im Studentenwohnheim. Natürlich war es anstrengend. Man musste zu Hause das Kind versorgen und sein Studium organisieren. Aber ich habe mich eben auch ganz rational und konzentriert organisiert. Schlendrian konnte ich mir nicht leisten. Ich habe plötzlich ganz anders angefangen zu studieren und ganz genau geschaut, wann ich was machen muss. Auf diese Weise habe ich sehr gut managen gelernt.

In den acht Wochen Sommerferien waren wir immer mit den Studentenbrigaden im sozialistischen Ausland unterwegs, nach Kasachstan, Bulgarien und so. Da gab es ein wildes Partyleben. Meine Mutter und meinen Vater haben wir überredet, dass sie den Enkel betreuen. Sie wohnen ja nicht in Berlin und waren auch willig, weil sie ihn dann mal da hatten. Ansonsten bin ich gar nicht der Partytyp, mehr der Theatertyp. In studentischen Zusammenhängen war ein Babysitter noch viel leichter zu organisieren als später ein professioneller Babysitter, den man bezahlen muss.

\section{Was empfehlen Sie Kolleginnen mit der Ambition, in die Wis- senschaft zu gehen?}

Eine Karriere in der Wissenschaft erfordert heute einen besonders langen Atem, viel länger als man ihn haben muss, wenn man Richterin oder Rechtsanwältin werden will. Das liegt primär daran, dass man eine Dissertation und Habilitation schreiben muss. Vor allem die langen Jahre der Habilitation, an der die Juristen trotz Juniorprofessur festgehalten haben, sind für Frauen problematisch. Sie fallen in die Zeit, in der man normalerweise die Kinder bekommt. Frauen müssen sich also damit auseinandersetzen, ob sie Zeit für das Kind opfern oder nicht, Karriere machen und dann auf die Lücke hoffen, wo das Kind hineinpasst. So hart sind die Fragen, denn für das eine wie für das andere kann es dann immer zu spät sein. Man ist nicht unbestimmt lange Zeit gebärfähig, genauso wenig wie man Wissenschaft erst später lernen kann, sondern nur in einer bestimmten Zeit. Es ist eine große Herausforderung und eine große Schwierigkeit. Dafür habe ich kein Rezept. Jede muss die Entscheidung für sich treffen.

\section{Ist es für Frauen in der Wissenschaft nach 1989 schwerer gewor- den oder sind die Hürden nur andere?}

Doch, es ist schwerer geworden, weil generell die Bedingungen für die Vereinbarkeit von Beruf und Familie schlechter geworden sind, als sie in der DDR waren. Die sehr speziellen Programme für Frauenförderung heute werden nicht so ernst genommen, wie sie in der DDR ernst genommen wurden. Natürlich war es eine staatliche Förderung von oben. Aber wenn Frauen diese genutzt haben, war es leichter, in diese Pfade reinzukommen. Heute setzt die ganze Quotierung immer voraus, dass man schon die passenden Qualifikationen erworben hat. Man muss fertig sein, um

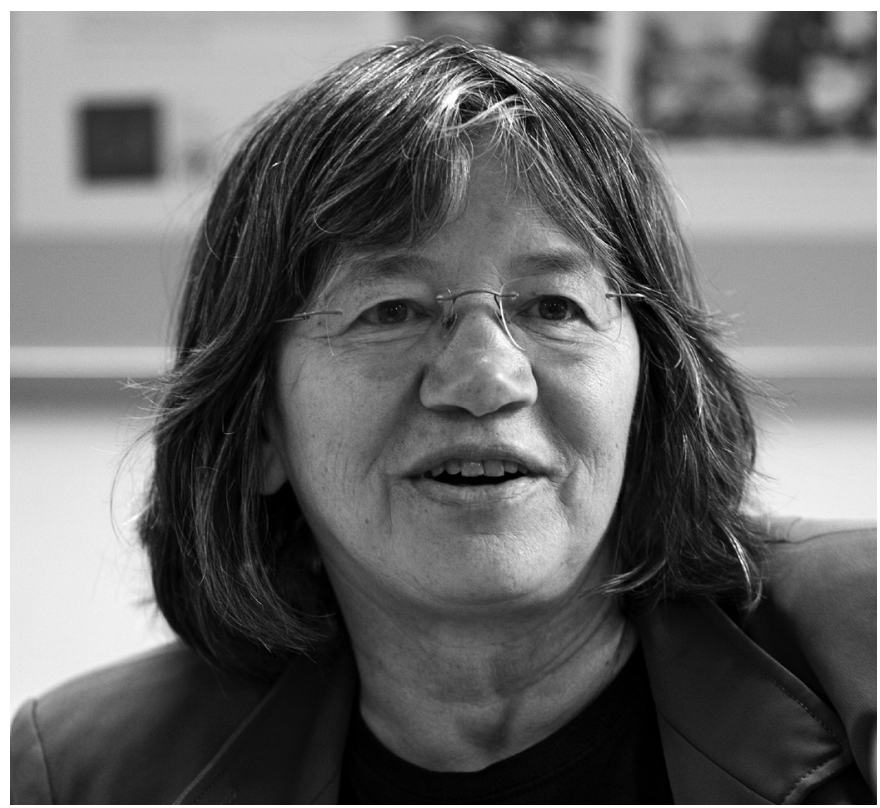

$\Delta$ Prof. Dr. Rosemarie Will, Humboldt-Universität zu Berlin (Foto: Sven Lüders)

dann über Quoten weitere Hürden zu überwinden, aber es fehlt die Unterstützung für den Weg zur Qualifikation. Kinderkriegen erfordert Zeit, die geht von der wissenschaftlichen Arbeit ganz klar ab. Mit der Rolle des Mannes als völlig gleichwertigem Elternteil alleine ist das nicht zu kompensieren. Es bleiben Schwangerschaft und Mutterschaft in einem Zeitraum, den der Mann nicht übernehmen kann. Die tradierten Bilder kommen noch dazu. Man findet nicht ohne Weiteres den Mann, der in vergleichbarer Weise bereit ist, die Karriere hinten anzustellen.

\section{Die Wiedervereinigung hat Ihr Berufsleben zweigeteilt. Wie haben Sie sie erlebt?}

Höchst unterschiedlich, ein großes Wechselbad der Gefühle und Auf und Ab. Als der Moment da war, als klar war, die Revolution kommt, sie hat Chancen, gab es eine ungeheure Euphorie. Was sich alles für Horizonte eröffneten. Doch am Anfang war es auch ganz bedrückend, dass es nicht so weiter geht. Dann löste sich der Knoten, alles schien ganz schnell zusammenzubrechen. Naiv, wie ich bin, hat die Euphorie bei mir lange angehalten. Man stellte sich vor, dass die Segnungen des Berufslebens im Westen einem nahtlos selbst zuteil werden. Ich bin noch lange mit diesem Gefühl der Euphorie herumgelaufen. Alle haben mich immer erschreckt angeguckt, wenn ich gesagt habe, ich bin Juristin. Dann hat es mich knallhart erwischt. Die juristische Fakultät wurde abgewickelt. Ich wurde evaluiert, meine erworbene Professur war weg und ich musste mich neu bewerben. Gleichzeitig musste ich vor die Studenten treten, die auch ihre Orientierung verloren hatten und von mir Ansprache erwarteten. Ich konnte ihnen nicht sagen, kümmere dich selbst um dich. Sie wollten ja mit mir reden. Ich war auf einmal in der Situation, Dinge zu erklären, die ich selber nicht wusste. Aber es hat mich in gewisser Weise gerettet, dass ich da vorne stand und sagen sollte, wie es geht, dass wir hier ohne Bücher lernen, wie die Fakultät abgewickelt wird, aber die Leute weiterstudieren, wie diese Fakultät erhalten bleiben soll. Das waren riesige Probleme, mit denen ich jeden Tag beschäftigt war, und die 
mich gezwungen haben, mich zu stellen und nicht zu sagen, wir machen hier alles zu. Ich war irgendwie verantwortlich und das war schwer. Ich habe Übergangsstudienordnungen für vier Jahrgänge gemacht und versuchte zu begreifen, was ein juristisches Curriculum im Westen ist und warum die sich so darüber streiten.

Es hat lange gedauert, bis ich durch diese Phase durch war. 1993 wurde ich für fünf Jahre berufen. Dann war ich zwei Jahre beim Bundesverfassungsgericht. Nach meiner Rückkehr lief meine Befristung ab und es ging darum, ob ich auf Lebenszeit bestellt werde. Der damalige CDU-Senator hat dies bis zum letzten Tag herausgezögert. Schließlich ging es darum, ob ich verbeamtet werde oder im Angestelltenverhältnis bleibe. Die eigene existentielle Unsicherheit kam mir endlos vor. 1998 wurde ich auf Lebenszeit angestellt das war die Variante für Ossis. Erst 2001 wurde ich verbeamtet. Zwischendurch hatte ich Phasen, wo ich aggressiv wurde und diese ganzen Faxen dicke hatte. Aber irgendwann wurde ich gelassener und habe auch gemerkt, dass Vieles zurück kam, was ich früher konnte und ich wieder ein Bild meiner Fähigkeiten hatte. Die zwei Jahre am Verfassungsgericht waren eine wunderbare Sache. Ich habe im Schnelldurchgang von der Pike auf Verfassungsrecht gelernt und hatte auch schon vorher mit den großen Verfassungsrechtlern Prof. Dr. Dieter Grimm und Prof. Dr. Bernhard Schlink, mit auch wirklichem Interesse an der deutschen Wiedervereinigung, wunderbare Unterstützer. Schlink kannte ich schon von der Expertenkommission des Runden Tisches. Er wurde später von Nordrhein-Westfalen freigestellt und arbeitete an der Humboldt-Universität. Grimm kam einfach aus Interesse, nachdem er gerade Verfassungsrichter geworden war. Es war Glück, dass nicht Leute geschickt wurden, die dann hier aus ideologischen Gründen aufräumen wollten. Selbst als dann jemand geschickt wurde, war es mit Hans Meyer jemand, der schon vorher hier unterrichtet hatte, ein anerkannter, sehr herausragender Verfassungsrechtler und Öffentlich-Rechtler und eben jemand, der auch liberal war und uns kannte.

Jeden Monat wurde ein Skandal über uns ausgebreitet. Ich bin im politischen Raum pausenlos angegriffen worden. Doch da ich immer Mitspieler hier hatte, mit denen ich sehr eng und sehr gut zusammen gearbeitet habe, habe ich mich gar nicht so vor diesen schrecklichen Presseberichten gefürchtet. Die Neuberufungen aus dem Westen waren exzellent. Das war ein großer Vorteil, den die Berliner Fakultät bzw. Universität insgesamt hatte.

\section{Hat sich Ihr Berufsleben radikal verändert oder einfach weiter- entwickelt?}

Ja, das hat es. Das Dasein als Hochschullehrerin änderte sich massiv mit dem Übergang in den Westen. Jura wurde über Nacht ein Massenfach, was es in der DDR nie war. Das bedeutet für einen Hochschullehrer, dass er plötzlich ganz entfernt ist von den Studenten. Alle kennen ihn, er kennt nur noch wenige. Die individualisierte Zusammenarbeit mit den Studenten gab es nicht mehr oder jedenfalls eher als Ausnahme denn als Regel. Der Hochschullehrer hat insgesamt eine ganz andere Rolle. In der DDR war er derjenige, der Bildung schlechthin vermittelte und die ganzen Ausfälle im Informationssystem im Zweifel ausgleichen musste. Wenn Sie über viele Dinge nicht informiert sind, findet das Gespräch viel stärker an den Universitäten statt. Heute ist man Lieferant für bestimmte
Dinge und nur in glücklichen Fällen orientieren die Leute sich an einem und fragen einen nicht nur um juristischen Rat.

Man hat natürlich viel mehr Freiheiten. Man kann alles lesen, was man will, aber man muss sich ständig entscheiden, was man liest und was nicht. In der DDR lief man jedem Buch hinterher und dann las man es natürlich. Als Professor hat man plötzlich einen Apparat, also studentische Mitarbeiter. Vorher gab es Assistenten, die in Instituten zusammengefasst und nur insofern jemand zugeordnet waren, als sie bei demjenigen promovierten.

\section{In den letzten Jahren waren Sie lange im Ausland. Haben Sie etwas nachzuholen?}

Ja. Ich habe alle Reisemöglichkeiten, die es in der DDR gab, ausgeschöpft. Das waren bekanntlich wenige, aber ich habe alles versucht, das sozialistische Ausland zu bereisen. Und ich habe es auch da schon gerne gemacht. Als die Grenzen aufgingen, habe ich das weiter ausgedehnt. Ich lasse gerne am Schreibtisch den Bleistift fallen, packe das Buch ein, schlage es anderswo wieder auf und arbeite dort weiter. Das habe ich inzwischen perfektioniert und kann manchmal nur noch schreiben, wenn ich wegfahre.

\section{Sie wurden anlässlich Ihrer Wahl zur Richterin am Verfassungs- gericht des Landes Brandenburg stark kritisiert. Wie sind Sie damit umgegangen?}

Ich habe mir gesagt, das bezieht sich nicht auf mich, sondern auf die Frage, wie man oder insbesondere auch die SPD mit Leuten umgeht, die Mitglied der SED waren und die in der DDR Karriere gemacht haben. Die SPD-Frauen haben mich vorgeschlagen. Regine Hildebrandt sah sich dann meinen Lebenslauf an und meinte, sagen Sie mal, Sie waren aber eine Streberin in der DDR. Ich antwortete, ja, klar, sieht man doch. Sie war cool genug, um zu reagieren mit, naja gut, das müssen ja nicht die schlechtesten Menschen gewesen sein, die da weiß ich was wollten. Dann gab es in der SPD-Fraktion im Landtag, die mich vorschlagen und durchsetzen musste, die Männer, die andere Männer aus dem Westen wollten. Die Kandidaten wurden vor die gesamte Fraktion geladen und vernommen - ich zwischen eineinhalb und zwei Stunden, meine beiden Konkurrenten jeweils für 20 bis 30 Minuten. Einer meiner Freunde kam einen Tag zuvor vorbei und wollte mich briefen, was ich zunächst für aussichtslos hielt. Er hatte aber über die wahrscheinliche Strategie meiner „speziellen Freunde" nachgedacht und überzeugte mich davon, meine Schriften aus der Wendezeit einzupacken. Tatsächlich schlugen diese während der Anhörung meine Schriften auf und zitierten daraus, etwa: „Da steht, dass man eine staatsfeindliche Opposition unter Umständen auch verbieten kann. Das haben Sie geschrieben, und jetzt wollen Sie Verfassungsrichterin werden? “ Aber ich war ja nun vorbereitet und habe sie aufgefordert weiterzulesen: „Man muss aber vorher freie Wahlen durchführen. " Und dann darüber zu reden. Es ging gar nicht darum, dass ich in der SED war oder was ich in der DDR war. Das war relativ stromlinienförmig, nichts Besonderes. Es ging vielmehr um meine Rolle im Übergang, gegen die insbesondere meine SPD-Genossen etwas hatten, weil ich zur 3. Weg-Plattform innerhalb der SED gehörte, die das Reformprogramm geschrieben und bei der Abstimmung im außerordentlichen Parteitag im Dezember 1989 über die Auflösung der SED verloren 
hatte. Mir wurde unterstellt, ich wolle die Konterrevolution. Darauf konnte ich immer nur entgegnen, das wollte ich ganz bestimmt nicht. Unser Programm wurde vom Neuen Forum abgeschrieben und die Vorwürfe waren daher gar nicht haltbar. Also haben sie krachend verloren. Nach diesen fast 2 Stunden Diskussion hat die Fraktion zu 80 Prozent für mich gestimmt. Der Landtag hat mich quer über alle Fraktionen bestätigt - auch in der CDU gab es Abtrünnige (die mich gewählt haben), ebenso bei der SPD und der PDS (die mich nicht gewählt haben, den bei den Linken galt ich nach meinem Austritt als Abtrünnige).

\section{Warum sind Sie djb-Mitglied geworden? Und 2011 haben Sie für den djb die Ausstellung „Juristinnen in der DDR“ konzipiert und realisiert.}

Adelhaid Brand hat mich gefragt, ob ich den DDR-Teil in „Juristinnen in Deutschland“ schreiben würde. Das habe ich gemacht. In dem Zusammenhang habe ich den djb wahrgenommen und bin eingetreten.

Die Ausstellung war ein Anliegen des djb an mich. Die Planung gab es schon länger, sie ging zurück auf eine schon existente Ausstellung über das Leben von Dr. Liselotte Kottler aus Schmalkalden. Es fehlte allerdings ein schlüssiges Konzept. Bei der Stange hat mich gehalten, dass es Leute gab, die diese Ausstellung verhindern wollten. Man muss bloß ausdrücklich sagen, dass man mich nicht will, dann finde ich Interesse an der Sache. Und das war hier der Fall. Es hat mich angespornt zu sagen, das kriege ich am Ende doch hin. Es ist ein ganz einfaches Verhaltensmuster. Wenn man es anwendet, dann funktioniere ich.
Sie engagieren sich umfangreich ehrenamtlich, insbesondere in der Humanistischen Union. Was ist Ihre Motivation?

Die Humanistische Union hat sich für die Arbeit am Runden Tisch interessiert und sie haben mich eingeladen. Sie sind sehr plural, ohne eine ideologische Festlegung - viele SPD-Mitglieder, Grüne, aber auch FDP, also klassische Liberale. Ich bin wahrscheinlich zu konservativ und zu verschreckt und will mich nicht mehr festlegen. Das bildungsbürgerliche Klima und der vornehm freundliche Umgang haben mich in meiner Heimatlosigkeit beeindruckt. Man hat mich nicht auf die Ossi-Position gestellt. Und bis heute finde ich diese bürgerrechtliche Perspektive auf juristische Probleme von besonderem Reiz. Deswegen mache ich da mit. Ich lerne unendlich viel. Ohne dass ich je Datenschützerin war, kenne ich alle großen datenschutzrechtlichen Probleme, weil ich sie als Bürgerrechtsprobleme kennengelernt habe. Das lässt sich mit meinem Fach gut verbinden und es macht mir intellektuell und politisch Spaß. Der Vorsitz war jedoch sehr anstrengend. Man ist dann für die Geschäfte zuständig, das nervt auch, kostet viel Zeit und lenkt von den fachlichen Fragen ab. Aber jetzt ist das vorbei und es ist o.k.

\section{Was machen Sie nun, nach Ihrer Emeritierung?}

Verzweifeln, weil ich nicht wirklich weiß, was ich zuerst machen soll. Und worauf ich mich jetzt festlegen soll. Ich muss erst mal sehen und ein Konzept entwickeln. Ich bin nicht so wirklich vorbereitet und haben weder Plan A noch B.

\section{Vielen Dank für das Gespräch!}

\section{Impressum}

Schriftleitung

Anke Gimbal, Rechtsassessorin (V.i.S.d.P.)

Katrin Lange

\section{Redaktionsanschrift}

Deutscher Juristinnenbund e. V.

Anklamer Str. 38

10115 Berlin

Telefon: 030 443270-0

Telefax: 030 443270-22

E-Mail: geschaeftsstelle@djb.de

www.djb.de

\section{Erscheinungsweise:}

4 Ausgaben im Jahr

\section{Bezugspreise 2014}

Jahresabonnement 54,- $€$; Einzelheft 15,- $€$. Alle Preise verstehen sich incl. MWSt, zzgl. Vertriebskostenanteil.

Bestellmöglichkeit

Bestellungen beim örtlichen Buchhandel oder direkt bei der Nomos Verlagsgesellschaft Baden-Baden

\section{Kündigungsfrist}

jeweils drei Monate vor Kalenderjahresende
Bankverbindung generell

Zahlungen jeweils im Voraus an Nomos Verlagsgesellschaft, Postbank Karlsruhe: IBAN: DE07 6601007500736367 51, BIC: PBNKDEFF oder Sparkasse Baden-Baden Gaggenau: IBAN: DE05 6625003000050022 66, BIC: SOLADES1BAD

\section{Druck und Verlag}

Nomos Verlagsgesellschaft $\mathrm{mbH} \&$ Co. KG Waldseestr. 3-5, D-76530 Baden-Baden Telefon (07221) 2104-O/Fax (07221) 2104-27 E-Mailnomos@nomos.de

\section{Anzeigen}

Sales friendly Verlagsdienstleistungen

Pfaffenweg 15, 53227 Bonn

Telefon (0228) 978980, Fax (o228) 9789820

E-Mail roos@sales-friendly.de

\section{Urheber- und Verlagsrechte}

Die Zeitschrift sowie alle in ihr enthaltenen einzelnen Beiträge und Abbildungen sind urheberrechtlich geschützt. Jede Verwertung, die nicht ausdrücklich vom Urheberrechtsgesetz zugelassen ist, bedarf der vorherigen Zustimmung des Verlags.

Mit der Annahme zur Veröffentlichung überträgt der Autor dem Verlag das ausschließliche Verlagsrecht für die Zeit bis zum Ablauf des Ur- heberrechts. Eingeschlossen sind insbesondere auch das Recht zur Herstellung elektronischer Versionen und zur Einspeicherung in Datenbanken sowie das Recht zu deren Vervielfältigung und Verbreitung online oder offline ohne zusätzliche Vergütung. Nach Ablauf eines Jahres kann der Autor anderen Verlagen eine einfache Abdruckgenehmigung erteilen; das Recht an der elektronischen Version verbleibt beim Verlag. Namentlich gekennzeichnete Beiträge geben nicht in jedem Fall die Meinung der Herausgeber/ Redaktion oder des Verlages wieder. Unverlangt eingesendete Manuskripte - für die keine Haftung übernommen wird - gelten als Veröffentlichungsvorschlag zu den Bedingungen des Verlages.

Die Redaktion behält sich eine längere Prüfungsfrist vor. Eine Haftung bei Beschädigung oder Verlust wird nicht übernommen. Bei unverlangt zugesandten Rezensionsstücken keine Garantie für Besprechung oder Rückgabe. Es werden nur unveröffentlichte Originalarbeiten angenommen. Die Verfasser erklären sich mit einer nicht sinnentstellenden redaktionellen Bearbeitung einverstanden.

Der Nomos Verlag beachtet die Regeln des Börsenvereins des Deutschen Buchhandels e.V. zur Verwendung von Buchrezensionen. ISSN 1866-377X 